\title{
RESONANCE AND IONIZATION POTENTIALS FOR ELECTRONS IN CADMIUM VAPOR
}

\author{
By John T. Tate, Assistant Physicist, and Paul D. Foote, Associate Physicist
}

The phenomena connected with the passage of electrons through mercury vapor have been fairly completely investigated. Franck and Hertz ${ }^{1}$ have shown that when the velocity of the electrons is less than that attained in falling through 4.9 volts there is no loss of energy at impact with a mercury atom. After falling through a difference in potential of 4.9 volts, however, the electrons are capable of transferring all of their kinetic energy to the mercury atom. The energy absorbed by the atom under these conditions is emitted as radiant energy of the single wave length, $\lambda=2536.72 \AA$. It was at first thought that this radiation of light was the result of a recombination with the atom of an electron which had been removed from it by the energy of impact. That this is not the case was proven by one of the writers, ${ }^{2}$ who showed that no appreciable ionization takes place in mercury vapor until the electrons have fallen through a difference in potential of 10.3 volts. Later Gaucher, ${ }^{3}$ using a method which was sensitive to secondary photo-electric effects obtained results which were thought to prove conclusively the setting in of ionization at 4.9 volts. The more recent work of Davis and Gaucher ${ }^{4}$ eliminates these secondary effects, and the value obtained for the ionizing potential, I 0.4 volts, is in good agreement with that already experimentally determined by Tate.

1 Franck and Hertz, Verh. d. Phys. Ges., 16, pp. 457-467, 1914.

2 Tate, Phys. R., 7, p. 686, r9r6; Phys. R., 10, p. 81, 19r7.

Gaucher, Phys. R., 8, p. 56r, 19r6.

- Davis and Gaucher, Phys. R., 10, D. 101, 1917. 
It seems evident, therefore, that the energy lost by an electron having a velocity corresponding to 4.9 volts on impact with a mercury atom goes over into energy of agitation of one of the electrons bound in the atom and that the natural frequency of vibration of this electron corresponds to a wave length of $2536.72 \AA$. To explain the large transfer of energy at such a collision, we may assume that the electron is traveling with a velocity such that it remains within reaction distance of the atom during a time which bears some simple relation to the natural period of vibration of one of the bound electrons. The velocity of the impinging electron for which this condition is true may be termed a resonance velocity of the electron in mercury vapor. That there may be other resonance velocities than that corresponding to 4.9 volts has been recently shown by Davis and Gaucher. ${ }^{5}$

It is now known that a real ionization of the mercury atom occurs at about 10.3 or 10.4 volts. This ionization is accompanied by the emission of the complete mercury spectrum. ${ }^{\circ}$

Franck and Hertz were the first to point out that the frequency of the radiation emitted at 4.9 volts is that which should follow from the well-known relation $h v=e V$, if we substitute 4.9 volts for $V$. McLennan has pointed out that the ionizing potential might be predicted on the basis of Bohr's theory by substituting for $v$ in the above relation the frequency $v=(1.5, S)$ of the mercury spectrum. The value so calculated is $10.4 \mathrm{I}$ volts (putting $h=$ $\left.6.56 \times 10^{-27}\right) .^{7}$ This value is in excellent agreement with the experimental values of 10.3 volts, obtained by Tate, and, more recently, by Davis and Gaucher, of I0.4 volts. From observations on the single and many lined spectra of other metallic vapors McLennan has been able to predict the resonance and ionization potentials for electrons in these vapors.

An experimental determination of the resonance and ionization potentials for electrons in cadmium vapor is the purpose of the present investigation.

The general method employed is the same as that used by one of the writers ${ }^{8}$ for determining the ionizing potential in mercury vapor. The arrangement of apparatus is shown in Fig. I. Cadmium was vaporized at the bottom of the pyrex glass tube, and the vapor, after passing through the superheated ionization chamber, condensed in the upper half of the tube. The ionization 
chamber was supported entirely by steel rods running from the top of the tube. The source of electrons was the hot platinum wire cathode $A$, of low resistance, coated with lime. Surrounding the cathode was a cylinder of nickel net, $B$, and outside and coaxial with this a cylinder of nickel foil, $C$. The apparatus was evacuated by means of a Langmuir condensation pump or by a Stimson ${ }^{9}$ two-stage condensation pump. In general, the pressure as registered by a McLeod gage was about $\mathrm{ro}^{-5} \mathrm{~cm} \mathrm{Hg}$. Sufficient vapor was obtained by heating the cadmium to its melting point.

The experimental procedure consisted in applying a constant retarding potential of about 2 volts between the net and the outside cylinder and measuring both the total current from the hot wire and that portion of it which reached the outside cylinder against the retarding field, as functions of a varying accelerating potential applied between the hot wire and net.

The results of the present work are represented graphically by Figs. 2 and 3. Reference numbers from $\mathrm{I}$ to 8 are assigned to the curves in these figures, and a complete analysis of the curves is given in Table $\mathrm{I}$. Curves $\mathrm{I}$ to 7 represent the current (in arbitrary

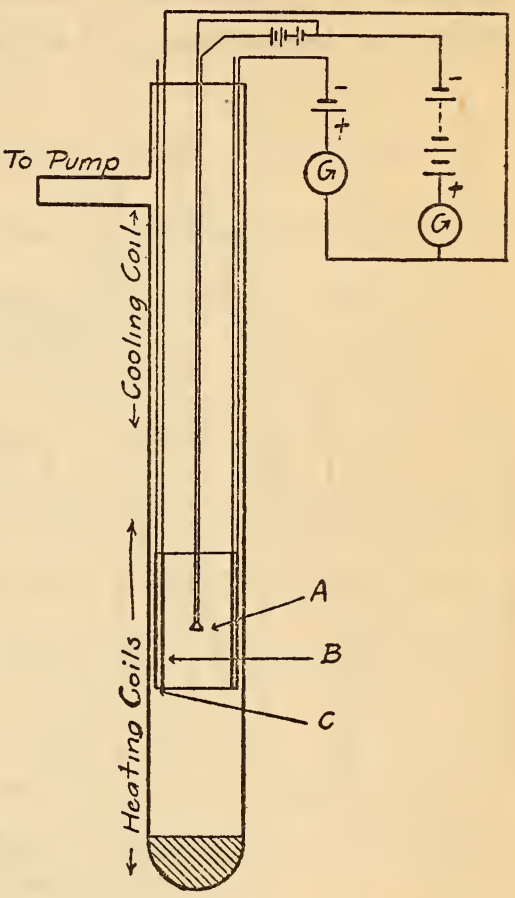

FIG. I.-Diagram of apparatus units) flowing between the net and outside cylinder of the ionization chamber as a function of the accelerating potential applied between the hot-wire cathode and the net. Curve 8 represents the total current from the hot wire as a function of the same accelerating potential.

The curves $\mathrm{I}$ to 7 are similar to those obtained by Franck and Hertz and by Tate for mercury vapor, in that they are characterized by successive maxima occurring at definite intervals. The potential difference between the successive corresponding parts of these maxima represents the potential through which an electron must fall in order that, on collision, it be capable of trans- 
ferring its kinetic energy to the cadmium atom. Curve 8 shows the characteristic variation with potential of the current from a hot wire in the presence of a gas or vapor at low pressure. The sudden increase in current at about 9 volts is due to ionization of the cadmium atom by impact with electrons having a velocity corresponding to that potential.

In the curves I to 6 , Fig. 2 , the points $a, b, c$ represent the voltages at which occurs a pronounced falling off in the rate of increase of current with potential. These points must be interpreted as determining the applied voltages at which the electrons

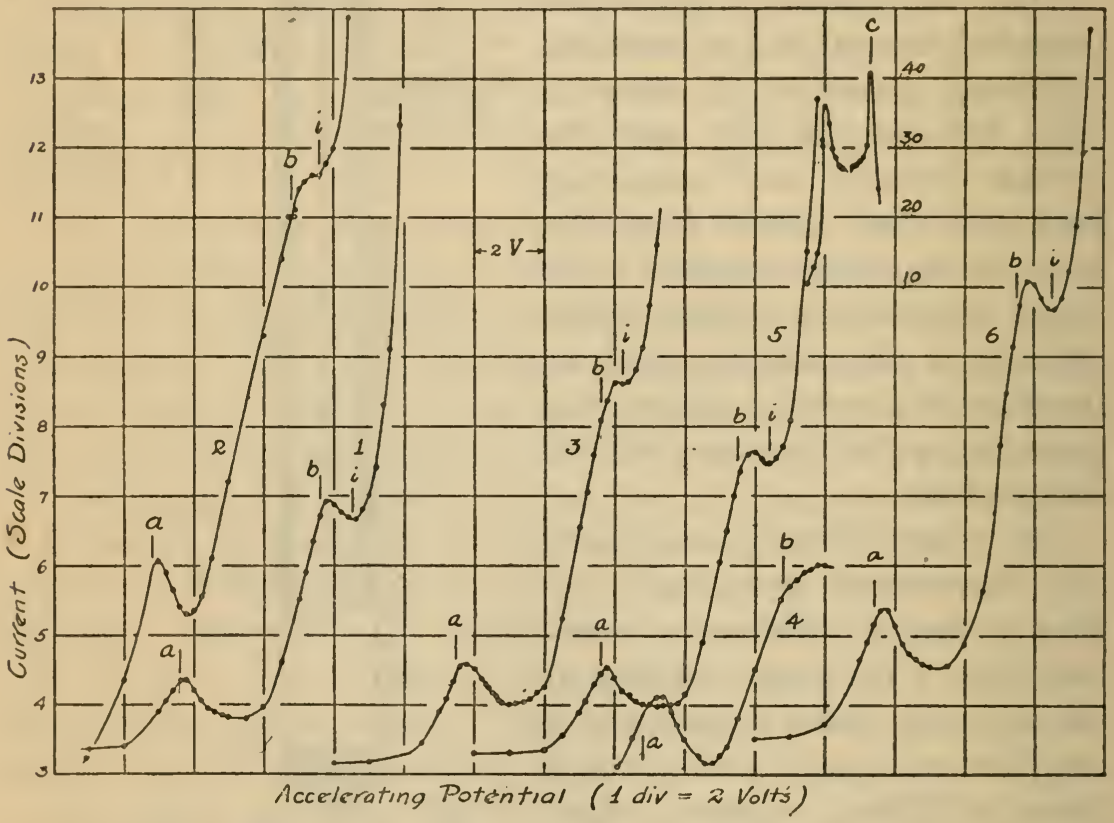

FIG. 2.-Variation with accelerating potential of current of outsidecylinder

begin to suffer inelastic collision. If the electrons were emitted from the hot cathode with zero velocity the potential of the points $a$ would represent the actual value of the resonance potential. Since, however, the electrons are ejected with some initial velocity, all potentials read from the curve must be corrected for the potential corresponding to the initial velocity. The value of the initial velocity is readily obtained from the curves. Points $a$ represent the resonance potential minus the initial potential; points $b$ represent twice the resonance potential minus the initial potential. Whence, the difference between $b$ and $a$ is the correct resonance potential, and the latter value together with the value $a$ gives the initial potential. A similar procedure may be carried through for 
the points of higher order, such as $c$, curve 5 , and also in curve 7 . Curve 7 is especially interesting. Resonance collision is observed to take place at five successive multiples of the fundamental potential interval, viz, at $a, b \quad c, d$, and $e$.

In addition to these points, all the curves show an abrupt change in curvature at $i$, followed by a very rapid increase in current. This fact, taken in connection with the abrupt change in totalcurrent curve 8 at $P$, coincident with $i$, indicates that the setting in of ionization, shown by $P$, produces a redistribution of potential

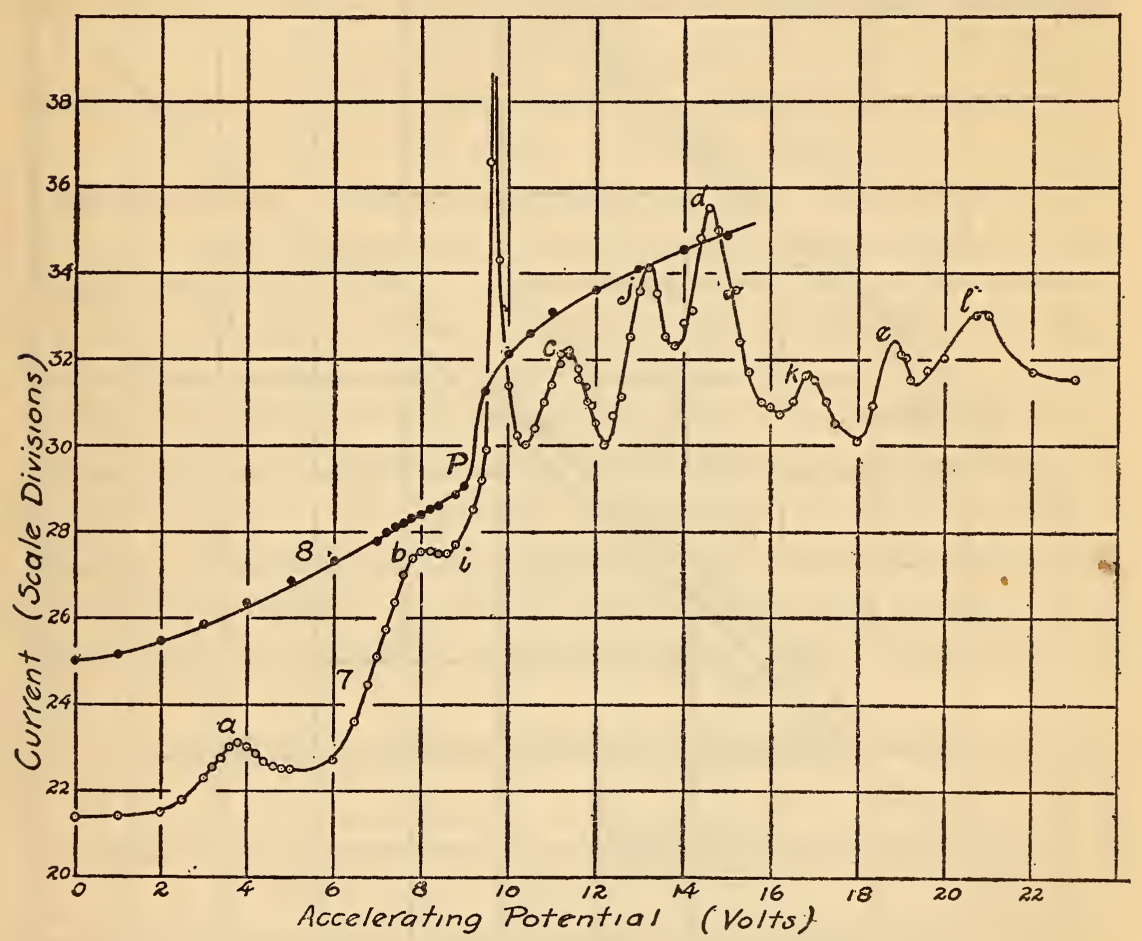

FIG. 3.-Variation with accelerating potential of current to outside cylinder and of total current from hot wire

between the wire and net, which is such as to result in a large increase in the number of electrons reaching the outside cylinder. The effect is rapidly counterbalanced, however, by the penetration and formation of positive ions in the space between the net and cylinder. The presence of inelastic impact at $P$ is indicated by the occurrence of three successive maxima (more accurately, pronounced changes in curvature as discussed above) at potentials differing from $P$ by multiples of the fundamental resonance potential, viz, at $j, k$, and $l$. These three points represent not 
only resonance collision of the electrons liberated from the atom with zero velocity at ionization, but also resonance collision of those electrons whose kinetic energy was lost in producing the ionization.

In determining the applied potential at which inelastic impact occurs, $a, b, c$, etc., were chosen at the points where the curves show a marked falling off in the rate of increase of current. Were there

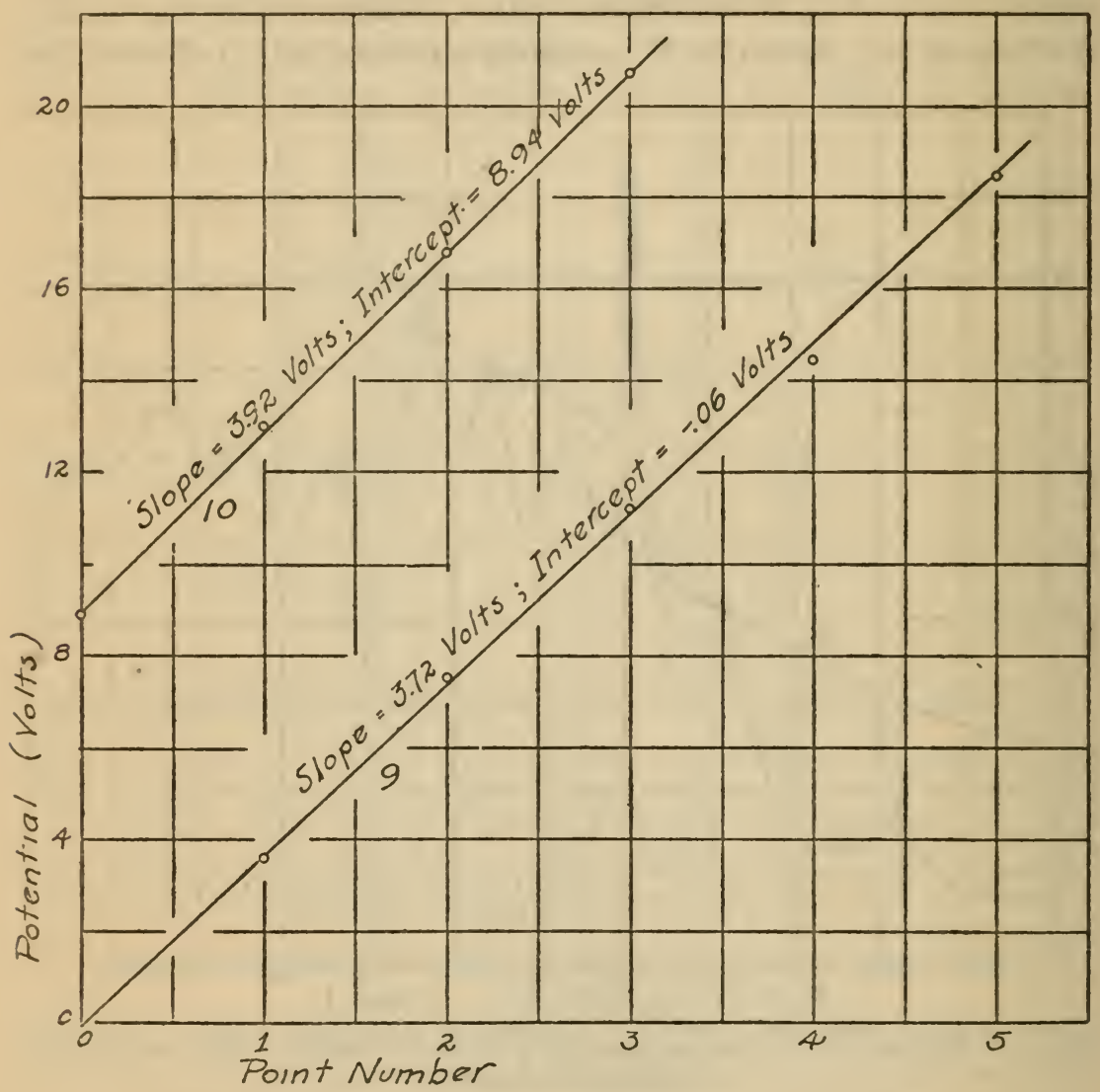

FIG. 4.-Graphical representation of data from curves 7 and 8

no inelastic collision at $a$, for example, the lower portion of the curve would continue to increase approximately exponentially. The curves were carefully plotted on a large scale and this portion of the curve graphically extrapolated. The point $a$ was chosen where the deviation of the extrapolated curve from the actual curve reached a predetermined very small amount. A similar procedure was adopted for the other points. Since one is here concerned only with differences between successive, similarly chosen points, 
there can be little question in regard to the somewhat arbitrary method of choice.

The method of least squares was applied to the data obtained from curves 7 and 8. If $y$ is the initial potential of the electrons, $x$ the resonance potential, and $Q$ the ionization potential, we have:

$$
\left.\left.\begin{array}{rl}
a=x-y \\
b=2 x-y \\
c=3 x-y \\
d=4 x-y \\
e=5 x-y
\end{array}\right\} \mathrm{I} . \quad \begin{array}{c}
Q=P+y \\
j=P+x \\
k=P+2 x \\
l=P+3 x
\end{array}\right\} \text { II. }
$$

The solution of these equations may be respresented graphically by Fig. 4. The slope of the curve 9 (group I, above) determines the resonance potential, and the intercept on the potential axis the initial potential. The slope of curve ro (group II, above) also determines the resonance potential and its intercept the most probable value of the point $P$ on the basis of equal weights to the observed points $P, j, k, l$. It is of interest to note that the new value for $P$ differs by a very small amount from the value obtained by graphical means, using only curve 8 .

The true value of the ionizing potential $Q$ is obtained from the relation $Q=P+y$, where $y$ is the initial velocity as determined by group I, above. The results of the present work are detailed in Table $\mathrm{I}$. Weighted averages give the most probable value of the resonance potential as 3.88 volts and the ionizing potential 8.92 volts, with an estimated probable error slightly less than o.I volts.

TABLE 1.-Resonance and Ionization Potentials for Cadmium

\begin{tabular}{|c|c|c|c|c|c|c|c|c|c|c|c|}
\hline \multirow{2}{*}{ Curve } & \multicolumn{5}{|c|}{ Resonance points } & \multirow{2}{*}{$\begin{array}{l}\text { Ioniza- } \\
\text { tion ob- } \\
\text { served. }\end{array}$} & \multirow{2}{*}{$\begin{array}{l}\text { Reso- } \\
\text { nance } \\
\text { poten- } \\
\text { tial }\end{array}$} & \multirow{2}{*}{ Weight } & \multirow{2}{*}{$\begin{array}{c}\text { Initial } \\
\text { velocity }\end{array}$} & \multirow{2}{*}{$\begin{array}{c}\text { Ioniza- } \\
\text { tion } \\
\text { poten- } \\
\text { tial }\end{array}$} & \multirow{2}{*}{ Weight } \\
\hline & 1st & $2 d$ & $3 d$ & 4th & 5th & & & & & & \\
\hline $1, \ldots \ldots \ldots \ldots \ldots \ldots \ldots$ & 3.6 & 7.6 & ..... & $\ldots \ldots$ & ...... & 8.5 & 4.0 & 1 & 0.9 & 8.9 & 1 \\
\hline $2 \ldots \ldots \ldots \ldots \ldots \ldots \ldots$ & 2.8 & 6.8 & & $\ldots$ & ....... & 7.6 & 4.0 & 1 & .8 & 8.8 & 1 \\
\hline $3 \ldots \ldots \ldots \ldots \ldots \ldots \ldots$ & 3.5 & 7.6 & & .. & ....... & 8.2 & 4.1 & 1 & .6 & 8.8 & 1 \\
\hline $4 \ldots \ldots \ldots \ldots \ldots \ldots \ldots$ & 2.8 & 6.8 & $\cdots$ & & & $\ldots \ldots$ & 4.0 & 1 & ..... & & . \\
\hline $5 \ldots$ & 3.6 & 7.5 & 11.3 & & & 8.4 & $\begin{array}{l}3.9 \\
3.8\end{array}$ & $\begin{array}{l}1 \\
1\end{array}$ & .9 & 8.6 & 1 \\
\hline $6 \ldots \ldots$ & 3.4 & 7.5 & ........ & $\ldots \ldots$ & ...... & 8.5 & 4.1 & 1 & 1.1 & 9.2 & 1 \\
\hline 7 (A) $\ldots$ & 3.6 & 7.7 & 11.2 & 14.4 & 18.4 & .......... & a 3.72 & 5 & $a .06$ & $\ldots \ldots$ & . \\
\hline $7(B)$ & 8.6 & 13.0 & 16.8 & 20.7 & ....... & $b 8.97$ & $b 3.92$ & 2 & $a .06$ & $b 9.03$ & 3 \\
\hline $8 \ldots \ldots$ & \multicolumn{5}{|c|}{ Total current curve........... } & 8.9 & ..... & -... & $a .06$ & $c 8.96$ & -. \\
\hline Mean & & & & & & & \multicolumn{3}{|c|}{3.88 volts. } & \multicolumn{2}{|c|}{8.92 volts. } \\
\hline
\end{tabular}

$c$ Not used in average because this point was involved in obtaining the value immediatcly above. 


\section{SUMMARY AND CONCLUSION}

The two types of electronic impact already mentioned in connection with mercury vapor are thus clearly shown to take place in cadmium vapor. The sudden decrease in current through the retarding field at an effective potential of 3.88 volts indicates a loss in energy of electrons having this critical velocity on impact with a cadmium atom. The fact that there is no corresponding increase in the total current at 3.88 volts shows that no appreciable ionization takes place at this potential. When the velocity of the electrons corresponds to 8.92 volts, the collisions again become inelastic, and the sudden increase in the total current indicates that the loss in energy of the colliding electrons has gone over into separating one or more of the electrons bound in the atom from the nucleus.

Under conditions such that only the first type of collision could take place McLennan and Henderson ${ }^{10}$ have shown that light of the single wave length $3260.17 \AA$ is emitted. That the entire spectrum of cadmium is probably radiated at potentials above 8.92 volts is indicated by the appearance of a visible glow around the hot wire at 9.2 volts. McLennan and Henderson were able to get the complete spectrum at about i 5 volts.

If we substitute the frequency corresponding to a wave length $3260.17 \AA$ in the relation $h v=e V$, we find the theoretical value for the resonance potential (putting $h=6.56 \mathrm{Io}^{-27} \mathrm{erg}$. sec.) to be 3.79 volts, as compared with the experimentally observed value 3.88 volts. If, following McLennan, we calculate the ionizing potential on the basis of Bohr's theory, using the frequency $v=(1.5, S)$ of the cadmium spectrum $(\lambda=1378.69 \AA)$ in the above relation, we obtain a theoretical value of 8.97 volts, as compared with 8.92 volts observed. The agreement is within the probable error of the experiment.

The results obtained for the resonance and ionization potentials for electrons in mercury and cadmium vapors must be regarded as another very striking example of the fundamental accuracy of conclusions based upon Bohr's theory of atomic structure. Especially is this true of the results for mercury vapor, since for a long time the view that ionization took place at 4.9 volts, together with the known fact that the radiation emitted at that potential was monochromatic, formed a very serious obstacle to the complete acceptance of the theory.

Washington, August 27, igi 7 . 